\title{
Consumption and Risk with hyperbolic discounting
}

\author{
Liutang Gong ${ }^{\mathrm{a}}$, William Smith ${ }^{\mathrm{b}, *}$, Heng-fu Zou ${ }^{\mathrm{c}, \mathrm{d}}$ \\ ${ }^{\text {a }}$ University of Beijing, China \\ ${ }^{\mathrm{b}}$ Department of Economics, Fogelman, United States \\ ${ }^{\mathrm{c}}$ University of Beijing, China and the World Bank, United States \\ d World Bank, United States
}

Received 18 June 2006; received in revised form 10 November 2006; accepted 20 December 2006

Available online 25 April 2007

\begin{abstract}
Hyperbolic discounting is not observationally equivalent to exponential discounting. It is always possible to calibrate an exponential model so that it predicts the same level of consumption as a hyperbolic model. However, the two models have radically different comparative statics.

(C) 2007 Elsevier B.V. All rights reserved.
\end{abstract}

Keywords: Consumption; Uncertainty; Hyperbolic discounting

JEL classification: D91; E21; G11

\section{Introduction}

The last decade has seen extensive research on dynamically inconsistent preferences [Ainslie and Haslam (1992), Laibson (1997a,b), Barro (1999), Bernheim et al. (1999), Gul and Pesendorfer (2001), Harris and Laibson (2001a,b, 2003), Krusell and Smith (2001)]. Only recently has there been any attempt to study the effects of dynamically inconsistent preferences on consumption and portfolio behavior under conditions of uncertainty. Palacios-Huerta (2003) has recently solved the savings problem for a version of Merton's (1969, 1971) classic model with constant relative risk aversion (CRRA) and hyperbolic discounting. He demonstrates that hyperbolic discounting raises consumption, so that a hyperbolic model

\footnotetext{
* Corresponding author. Tel.: +1 901678 3675; fax: +1 9016785380.

E-mail address: wtsmith@memphis.edu (W. Smith).
} 
is observationally equivalent to a model with exponential discounting, but with a higher discount rate. He infers that the canonical model remains "intact" after introducing hyperbolic discounting. This mirrors Barro's (1999) argument that the neoclassical growth model remains "intact" after introducing a nonconstant rate of time preference.

Our purpose in this paper is to make precise the sense in which hyperbolic discounting leaves the canonical model intact, and to explain the ways in which it does not. It is true that the level of consumption in a model with hyperbolic discounting is observationally equivalent to the level of consumption in a model of exponential discounting. However, hyperbolic discounting endogenizes the rate of time preference, making it depend in a non-linear way upon the expected growth in wealth. Thus the comparative statics of consumption under hyperbolic discounting are strikingly different from those under exponential discounting.

Consider how portfolio risk affects consumption. We demonstrate that the marginal effect of risk on consumption is always greater under hyperbolic discounting than under exponential discounting. Furthermore, hyperbolic discounting causes consumption to be a concave function of risk, while in the exponential benchmark the response is linear.

To understand these results it is useful to elaborate upon the intuitive story told by Harris and Laibson (2001a, p. 936). Suppose, for example, that relative risk aversion is less than one. Now imagine an increase in portfolio risk. In the exponential model, the investor responds by raising consumption. This is still true in the hyperbolic model. Now, however, the "current" self anticipates that the "future" self will be impatient and consume "too much." He realizes that the future self will increase consumption even more in response to the increase in risk, so he attaches less value to future consumption at the margin. Therefore, the increase in risk lowers the current self's discount factor on future consumption. This causes his current consumption to increase by more than in the exponential model. In other words, hyperbolic discounting amplifies the marginal effect of risk on consumption. However, this effect weakens as risk gets bigger, so that consumption increases at a decreasing rate as risk increases.

Harris and Laibson (2001b) and Luttmer and Mariotti (2003) also treat saving decisions in continuoustime with risky assets and hyperbolic discounting. Neither investigates the comparative statics of consumption.

\section{Consumption and portfolio choice with hyperbolic discounting}

We briefly recapitulate Palacios-Huerta's (2003) model. The consumer maximizes expected lifetime utility over an infinite planning horizon. There is quasi-hyperbolic discounting: starting at time $t$ the consumer's discount function decays exponentially at the constant rate $\beta$ until time $t+h$; just prior to time $t+h$ it drops discontinuously to a fraction $\delta \in(0,1]$ of its value, and then continuous to decay at the rate $\beta$. In other words, the discount function is

$$
\begin{aligned}
& e^{-\beta s}, t \leq s \leq t+h, \\
& \delta e^{-\beta s}, t+h \leq s<\infty .
\end{aligned}
$$

Following Merton (1969, 1971), the consumer's preferences are time-separable and the felicity function is CRRA. His expected lifetime utility is then

$$
E_{t} U_{t}=E_{t} \int_{t}^{t+h} e^{-\beta s} \frac{c_{s}^{1-b}}{1-b} \mathrm{~d} s+\delta \int_{t+h}^{\infty} e^{-\beta s} \frac{c_{s}^{1-b}}{1-b} \mathrm{~d} s
$$


Intuitively, the current self makes decisions from time $t$ to time $t+h$, when the next self takes charge. Setting $\delta=$ in Eq. (2) recovers the exponential preferences used by Merton $(1969,1971)$.

The consumer can invest in two assets. The riskless asset pays a constant rate of return $r$, while the price $P_{t}$ of the risky asset follows a geometric Brownian motion,

$$
\frac{\mathrm{d} P_{t}}{P_{t}}=\mu \mathrm{d} t+\sigma \mathrm{d} Z_{t},
$$

where $Z_{t}$ is a Wiener process. If $\theta_{t}$ is the share of wealth $W_{t}$ invested in the risky asset, then the consumer's budget constraint is

$$
\mathrm{d} W_{t}=\left\{\left[\left(1-\theta_{t}\right) r+\theta_{t} \mu\right] W_{t}-c_{t}\right\} \mathrm{d} t+\sigma \theta_{t} W_{t} \mathrm{~d} Z_{t} .
$$

The consumer chooses policies $\theta_{t}$ and $c_{t}$ to maximize Eq. (2) subject to Eq. (4), and given initial wealth $W_{0}$ Palacios-Huerta (2003) solves this problem to arrive at the following optimal policies. The portfolio demand is exactly the same as in Merton $(1969,1971)$ :

$$
\theta_{t}^{*}=\frac{\mu-r}{b \sigma^{2}} .
$$

Hyperbolic discounting has no effect on portfolio demands.

The consumption function is

$$
c_{t}^{*}=\alpha_{\mathrm{H}} W_{t} .
$$

The marginal propensity to consume (MPC) $\alpha_{\mathrm{H}}$ is determined implicitly by the equation

$$
\alpha_{\mathrm{H}}=\frac{\left\lfloor\beta+(1-\delta) \alpha_{\mathrm{H}} e^{-\beta h} E_{0}\left(W_{h} / W_{0}\right)^{1-b}\right\rfloor-(1-b)\left[\mu_{w}-b \sigma_{w}^{2} / 2\right]}{b},
$$

where $\mu_{w}=\left(1-\theta^{*}\right) r+\theta^{*} \mu$ and $\sigma_{w}^{2}=\theta^{* 2} \sigma^{2}$ are the optimal mean and variance of the rate of return to the portfolio. The subscript "H" denotes "hyperbolic," to distinguish it from the exponential benchmark. ${ }^{1}$ It is important to note that $E_{0}\left(W_{h} / W_{0}\right)^{1-b}$ is endogenous; its value will be given by Eq. (9) below.

Consider the properties of this consumption function. The second term in braces in Eq. (7) is the certainty equivalent rate of return to the portfolio. The response of consumption to changes in the certainty equivalent rate of return is governed by the magnitude of risk aversion, $b$.

The first term in braces is the "effective" rate of time preference. In the absence of hyperbolic discounting $(\delta=1)$ the rate of time preference would reduce to $\beta$ and we would recover the marginal propensity to consume in Merton $(1969,1971)$ :

$$
\alpha_{\mathrm{M}}=\frac{\beta-(1-b)\left[\mu_{w}-b \sigma_{w}^{2} / 2\right]}{b} .
$$

\footnotetext{
${ }^{1}$ For small $h, \alpha_{\mathrm{H}}>0$ requires $b>1-\delta$. The optimal policies must also satisfy a transversality condition. All derivations and technical details are in an appendix, available upon request.
} 
The subscript "M" stands for "Merton." Notice that in this case the MPC is a linear function of the certainty equivalent rate of return.

Comparing Eqs. (7) and (8), it is evident that hyperbolic discounting $(\delta<1)$ increases the effective rate of time preference from $\beta$ to $\beta+(1-\delta) \alpha e^{-\beta b} E_{0}\left(W_{h} / W_{0}\right)^{1-b}$. As explained by Harris and Laibson (2001a), the current self anticipates that the future self will consume too much and so attaches less value at the margin to future consumption. It follows that hyperbolic discounting raises consumption. Palacios-Huerta (2002) infers that hyperbolic discounting is observationally equivalent to exponential discounting, but with a higher discount rate.

But the marginal propensity to consume defined by Eq. (7) warrants closer scrutiny. Note that the rate of time preference with hyperbolic discounting depends upon the expected growth in wealth between period 0 and period $h$. Since wealth is log-normal, it is straightforward to calculate

$$
E_{0}\left(W_{h} / W_{0}\right)^{1-b}=e^{(1-b)\left[\mu_{w}-\alpha_{\mathrm{H}}-b \sigma_{w}^{2} / 2\right] h} .
$$

Changes in the mean and the variance of the rate of return, as well as changes in the MPC itself, alter the effective rate of time preference exponentially. This elucidates the non-linear way in which hyperbolic discounting affects time preference, and suggests that the comparative statics of consumption will be much richer than in the canonical model with exponential discounting.

\section{Risk and consumption}

How does uncertainty affect consumption in the presence of hyperbolic discounting? Since the portfolio demand is unaffected by discounting, we will abstract from the portfolio decision entirely in addressing this question: Henceforth, we assume there is no riskless asset. In this case, the marginal propensity to consume is determined implicitly by

$$
\alpha_{\mathrm{H}}=\frac{\left\lfloor\beta+(1-\delta) \alpha_{\mathrm{H}} e^{-\beta h+(1-b)\left[\mu-\alpha_{\mathrm{H}}-b \sigma^{2} / 2\right] h}\right\rfloor-(1-b)\left[\mu-b \sigma^{2} / 2\right]}{b} .
$$

The rate of time preference is an increasing function of the MPC.

To understand the comparative statics of the model with hyperbolic discounting, it is helpful to recall the MPC for the benchmark case with exponential discounting in Eq. (8). In that case consumption will increase or decrease with risk depending upon whether relative risk aversion is less than or greater than one,

$$
\partial \alpha_{\mathrm{M}} / \partial \sigma^{2}=(1-b) / 2
$$

The key thing to notice is that under exponential discounting consumption is a linear function of $\sigma^{2}$.

Now consider the effects of a change in risk on the MPC in the general case with hyperbolic discounting. Differentiating Eq. (10) we find

$$
\frac{\partial \alpha_{\mathrm{H}}}{\partial \sigma^{2}}=(1-b) \frac{b}{2} \frac{1-(1-\delta) h \alpha_{\mathrm{H}} e^{-\beta h} E_{0}\left(W_{h} / W_{0}\right)^{1-b}}{b+\left[(1-b) \alpha_{H} h-1\right](1-\delta) e^{-\beta h} E_{0}\left(W_{h} / W_{0}\right)^{1-b}} .
$$

It is still true that the sign of the effect of risk on consumption hinges upon the degree of risk aversion. However, the magnitude of this effect is no longer constant, and depends upon the importance of 


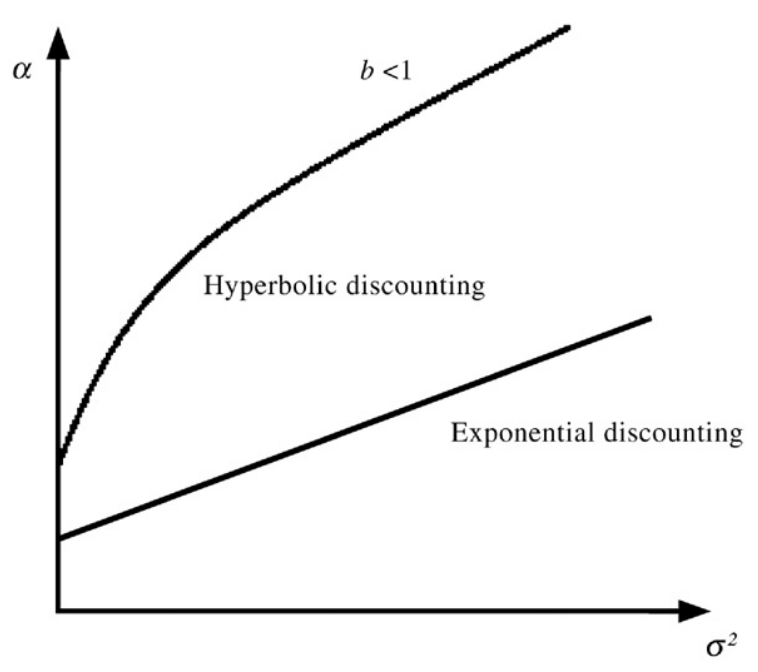

Fig. 1.

hyperbolic discounting $(\delta)$. Hyperbolic discounting disrupts the simple linear effect of risk on consumption with CRRA utility.

\section{How does hyperbolic discounting alter the comparative statics of risk?}

Proposition. The absolute value of the marginal effect of risk on consumption is greater under hyperbolic discounting than under exponential discounting: $\left|\partial \alpha_{H} / \partial \sigma^{2}\right|>\left|\partial \alpha_{M} / \partial \sigma^{2}\right|$. Furthermore, if $h$ is sufficiently small and $b>1-\delta$ consumption is a concave function of risk when discounting is hyperbolic: $\partial^{2} \alpha_{H} / \partial \sigma^{2{ }^{2}}<0 .{ }^{2}$

In other words, hyperbolic discounting amplifies the effect of risk on consumption, relative to the exponential benchmark. Consumption still increases or decreases with risk depending upon the magnitude of relative risk aversion. Now, however, it increases or decreases at a decreasing rate, rather than at a constant rate.

Figs. 1 and 2 depict consumption as a function of risk for the cases where $b$ is less than or greater than unity. The linear functions depict the exponential benchmarks. Notice that in either case consumption with hyperbolic discounting exceeds consumption under exponential discounting.

First suppose that $b<1$ shown in Fig. 1. If discounting is hyperbolic, then consumption is an increasing, concave function of risk. The hyperbolic consumption function is always steeper than the exponential, so that the marginal effect of risk on consumption is greater under hyperbolic discounting than under exponential discounting. However, the marginal impact of risk on consumption decreases as risk increases. The intuition for this behavior is quite simple. Compare the change in the MPC under exponential discounting in Eq. (11) with the change in the MPC under hyperbolic discounting in Eq. (12). Recall that under exponential discounting the rate of time preference is just $\beta$ while under hyperbolic discounting the "effective" rate of time preferences is $\beta+(1-\delta) \alpha e^{-\beta b} E_{0}\left(W_{h} / W_{0}\right)^{1-b}$ With exponential discounting the rate of time preference is constant, so the MPC increases linearly with risk. Under hyperbolic discounting, however, the increase in the MPC feeds back to raise the effective rate of time

\footnotetext{
2 The concavity of $\alpha_{\mathrm{H}}$ holds for small $h$, but not for the case of instantaneous gratification, when $h=0$ In most applications, however, the consumer presumably has some ability to commit to decisions over short periods.
} 


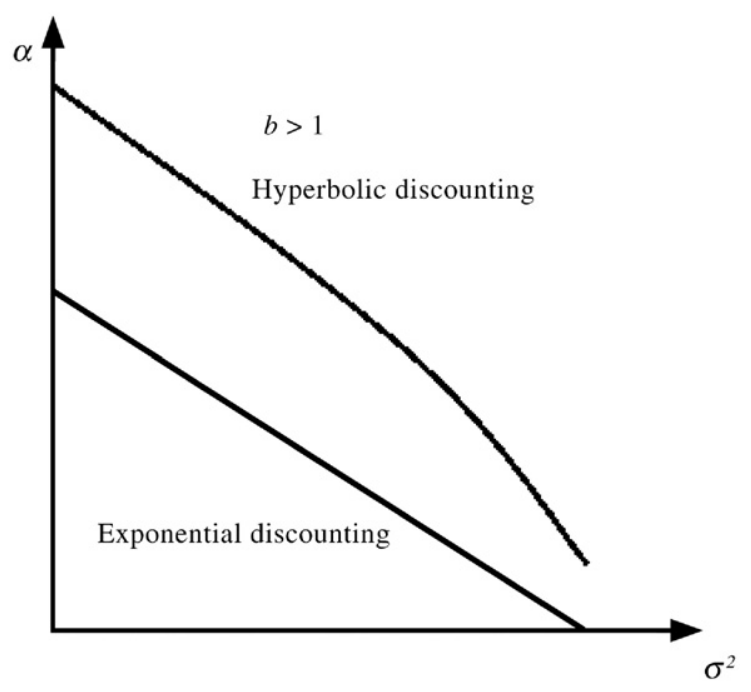

Fig. 2.

preference. This tends to magnify the increase in consumption caused by the increase in risk. However, the increase in risk also tends to lower the effective rate of time preference (for a given $\alpha_{\mathrm{H}}$ ) when $b<1$ [see Eq. (9)]. This exerts a dampening effect on the MPC which increases as risk increases in magnitude.

Conversely, suppose that $b>1$ In this case consumption is decreasing and concave in risk, as shown in Fig. 2. The slope of the hyperbolic curve is always more negative than for the exponential curve. Here, hyperbolic discounting amplifies the decrease in consumption caused by an increase in risk.

\section{Conclusion}

By endogenizing the rate of time preference, hyperbolic discounting amplifies the effect of changes in risk on consumption. This offers a broad warning about the extent to which "standard" models seem to remain "intact" in the face of hyperbolic discounting. The standard model is observationally equivalent to the hyperbolic model in the sense that the former can always be calibrated to match the consumption predicted by the latter. However, this does not imply that the comparative static predictions of the two models are the same.

\section{Appendix A. Consumption and risk with hyperbolic discounting}

\section{A.1. The transversality condition and some essential inequalities}

The optimal policies must satisfy the transversality condition (TVC)

$$
\lim _{t \rightarrow \infty} E b e^{-\beta t} W_{t}^{1-b}=0 .
$$

As in Merton $(1969,1971)$ the feasibility condition $\alpha_{\mathrm{H}}>0$ is a sufficient condition for the TVC to be satisfied. The TVC in turn implies that $e^{-\beta h} E_{0}\left(W_{h} / W_{0}\right)^{1-b}<1$ Thus, it also follows that $1-(1-\delta) h \alpha_{H} e^{-\beta h} E_{0}$ $\left(W_{h} / W_{0}\right)^{1-b}>0$. 
Notice that for small $h, h \alpha_{\mathrm{H}}<1$ Notice also that [from Eq. (7) in the text] for small $h$ it must be true that $b>1-\delta$ in order for $\alpha_{\mathrm{H}}>0$. Assuming that $b>1-\delta$ it then follows that, $b+\left[(1-b) \alpha_{H} h-1\right](1-\delta) e^{-\beta h} E_{0}$ $\left(W_{h} / W_{0}\right)^{1-b}>0$ for sufficiently small $h$.

\section{A.2. Existence and uniqueness of the MPC}

Write the right-hand side of Eq. (8) as RHS( $\alpha)$ The TVC implies RHS $(0)>0$ Furthermore, since $b+[(1-b)$ $\alpha h-1](1-\delta) e^{-\beta h} E_{0}\left(W_{h} / W_{0}\right)^{1-b}>0$, it can be shown that $0<\mathrm{dRHS} / \mathrm{d} \alpha<1$ Therefore, RHS crosses the $45^{\circ}$ line once.

\section{A.3. Proof of the proposition}

The first statement follows from comparing Eqs. (11) and (12) and using the fact that $1>\alpha_{\mathrm{H}} h$. The second statement follows from differentiating Eq. (12):

$$
\frac{\partial^{2} \alpha_{\mathrm{H}}}{\partial \sigma^{2^{2}}}=(1-b) b \frac{(1-\delta) h e^{-\beta h}}{2} \Omega,
$$

where

$$
\Omega=\frac{\left[(1-\delta) e^{-\beta h} E_{0}\left(W_{h} / W_{0}\right)^{1-b}-1\right] E_{0}\left(W_{h} / W_{0}\right)^{1-b} h \frac{\partial \alpha_{H}}{\partial \sigma^{2}}-(1-b)(1-h \alpha)\left[\frac{\partial \alpha_{H}}{\partial \sigma^{2}}+\frac{b}{2}\right]}{\left\{b+\left[(1-b) \alpha_{H} h-1\right](1-\delta) e^{-\beta h} E_{0}\left(W_{h} / W_{0}\right)^{1-b}\right\}^{2}} .
$$

Again, $1>h \alpha_{\mathrm{H}}$ for small $h$. The transversality condition implies that the first term in braces is negative.

Now consider the two cases mentioned in the Proposition. If $b<1$ then $\partial \alpha_{\mathrm{H}} / \partial \sigma^{2}>0$, so $\Omega<0$. Therefore $\partial^{2} \alpha_{\mathrm{H}} / \partial \sigma^{2}<0$. If $b>1$ then $\partial \alpha_{\mathrm{H}} / \partial \sigma^{2}<0$. If the last term in braces is positive then $\Omega>0$. It is easy to show, however, that this expression is positive if and only if $1>h \alpha_{\mathrm{H}}$. Therefore, if $b<1$ then $\partial^{2} \alpha_{\mathrm{H}} /$ $\partial \sigma^{2^{2}}<0$.

\section{References}

Ainslie, G., Haslam, N., 1992. Hyperbolic discounting. In: Loewenstein, G., Elster, J. (Eds.), Choice Over Time. Russell Sage Foundation, New York, pp. 57-92.

Barro, R., 1999. Ramsey meets laibson in the neoclassical growth model. Quarterly Journal of Economics 114, 1125-1152.

Bernheim, D., Ray, D., Yeltekin, S., 1999. Self-Control, Saving, and the Low Asset Trap, Working Paper, Stanford University. Gul, F., Pesendorfer, W., 2001. Temptation and self-control. Econometrica 69, 1403-1435.

Harris, C., Laibson, D., 2001a. Dynamic choices of hyperbolic consumers. Econometrica 69, 935-957.

Harris, C., Laibson, D., 2001b. Instantaneous Gratification, Working Paper, Harvard University.

Harris, C., Laibson, D., 2003. Hyperbolic discounting and consumption. In: Dewatripont, M., Hansen, L., Turnovsky, S. (Eds.), Advances in Economics and Econometrics: Theory and Applications, Eight World Congress, vol. I. Cambridge University Press, Cambridge.

Krusell, P., Smith, A., 2001. Consumption and Savings Decisions with Quasi-Geometric Discounting, Working Paper, Carnegie Mellon University.

Laibson, D., 1997a. Golden eggs and hyperbolic discounting. Quarterly Journal of Economics 112, $443-478$.

Laibson, D., 1997b. Hyperbolic Discount Functions and Time Preference Heterogeneity, Working Paper, Harvard University. 
Luttmer, E., Mariotti, T., 2003. Subjective discounting in an exchange economy. Journal of Political Economy 111, 959-989. Merton, R.C., 1969. Lifetime portfolio selection under uncertainty: the continuous time case. Review of Economics and Statistics $51,247-257$.

Merton, R.C., 1971. Optimum consumption and portfolio rules in a continuous-time model. Journal of Economic Theory 3, 373-413.

Palacios-Huerta, I., 2002. Consumption and Portfolio Rules Under Hyperbolic Discounting, Working Paper, Brown University. Palacios-Huerta, I., 2003. Consumption and Portfolio Rules Under Hyperbolic Discounting, Working Paper, Brown University. 